\title{
LA EVALUACIÓN FORMATIVA Y COMPARTIDA DESDE UN ENFOQUE COMPETENCIAL. APLICACIÓN PRÁCTICA EN TAREAS DE AULA EN PRIMARIA Y SECUNDARIA
}

Formative and shared assessment from a competence approach. Practical application for classroom tasks in primary and secondary education

A avaliação formativa y partilhada a partir de uma abordagem competencial. Aplicação prática em tarefas de sala de aula do ensino primário, básico e secundário

\author{
Carlos Heras Bernardino (1) \\ Israel Herrán Álvarez (2) \\ Ángel Pérez Pueyo (3)
}

(1) IES Prado de Santo Domingo, Alcorcón. Teléfono: +34916439100. Correo electrónico: carlos.herasbernardino@educa.madrid.org

(2) IES Doctor Sancho de Matienzo. Villasana de Mena. Burgos. Teléfono: +34665016492. Correo electrónico: israherran@gmail.com

(3) Universidad de León, España. Teléfono: +34 987293024. Correo electrónico: angel.perez.pueyo@unileon.es

\section{Resumen}

En el presente taller se analiza la importancia de la evaluación formativa en el desarrollo de las competencias clave. Para ello se describen dos propuestas de aula vinculadas al aprendizaje de contenidos eminentemente conceptuales desde una perspectiva participativa del alumnado, que centran su desarrollo en dos ejes fundamentales: 1) La integración de los procesos de evaluación formativa y compartida, y 2) La contribución al desarrollo de las competencias clave a partir de la secuenciación propuesta por el Proyecto INCOBA del Grupo Actitudes. Se presentan dos ejemplos, a) el diseño colaborativo de preguntas de examen y b) la búsqueda documental colaborativa combinada de la elaboración de un trabajo síntesis de 1000 palabras.

Palabras clave: Competencias clave; evaluación formativa; tareas competenciales; INCOBA 


\begin{abstract}
This workshop analyses the relevance of formative assessment in the development of the students' key competencies. To this aim, we will describe two proposals at the classroom level, which are related to the learning of (mainly) conceptual contents from a collaborative perspective. These proposals focus on two main axes: 1) The integration of formative and shared assessment processes within the learning process; and 2) The contribution to the development of key competences, on the basis of the sequencing proposed by the INCOBA Project, developed by the Grupo Actitudes. Two examples will be presented, a) the collaborative design of exam questions; and b) the combined collaborative documentary search for the elaboration of a 1000-word synthesis work.
\end{abstract} Keywords: Key competencies; formative assessment; competence tasks; INCOBA

\title{
Resumo
}

No presente workshop analisa-se a importância da avaliação formativa no desenvolvimento de competências chave. Para isso descrevem-se duas propostas de aula associadas à aprendizagem de conteúdos eminentemente concetuais a partir de uma perspetiva participativa do aluno, que centra o seu desenvolvimento em dois eixos fundamentais: 1) a integração dos processos de avaliação formativa e partilhada, y 2) a contribuição para o desenvolvimento das competências chave a partir da sequência proposta por o projeto INCOBA do grupo Actitudes. Apresentam-se dois exemplos, a) o desenho em colaboração de perguntas de exame y b) busca documental colaborativa e combinada da elaboração de um trabalho de síntese de 1000 palavras.

Palavras-chave: Competências chave; avaliação formativa; tarefas de competência; INCOBA

\section{Introducción}

El presente taller pone el acento en el papel fundamental que ejercen los procesos de evaluación formativa y compartida en el desarrollo de las competencias clave. Para ello, se presentan dos propuestas de aula que tienen como finalidad abordar de manera significativa y funcional el aprendizaje de contenidos curriculares que presentan una mayor carga teórica y conceptual, integrando en el proceso aprendizajes de carácter competencial.

La evaluación formativa y compartida desde un enfoque competencial. Aplicación práctica en tareas de 
La primera de las experiencias (Pérez-Pueyo, 2013; Lozano, Casado \& PérezPueyo, 2017) se centra en el uso formativo de las pruebas escritas, para lo cual nos alejamos de las metodologías tradicionales de exposición magistral para aproximarnos a las propuestas de carácter cooperativo. La segunda experiencia (Heras-Bernardino \& Pérez-Pueyo, 2017) muestra cómo poner los contenidos de cualquier materia al servicio del desarrollo de tres aspectos competenciales fundamentales: (1) Búsqueda y filtrado de información, (2) Gestión de trabajos colaborativos, y (2) Elaboración de un trabajo de síntesis.

\section{Propuesta 1: Diseño colaborativo de preguntas de examen.}

En el taller se intenta demostrar cómo es posible mejorar la actitud y los resultados de aprendizaje de una prueba escrita, en especial en aquellos alumnos que evidencian mayores reticencias y dificultades, intentado sacarle todo su potencial formativo gracias a la inclusión de la evaluación formativa y tareas de carácter competencial. La propuesta consta de tres tareas claramente diferenciadas: (a) la elaboración de preguntas de examen por parte de los alumnos, (b) concurso de repaso intergrupal y (c) la participación en dinámicas de coevaluación y autoevaluación tras el examen.

La primera tarea consta de una primera actividad en la que los alumnos, organizados en grupos de entre 3 a 5 miembros, trabajan sobre la documentación aportada por el profesor para redactar un conjunto de preguntas con sus respectivas respuestas $^{1}$ para un examen posterior. Para ayudar en este trabajo colaborativo, se incorporan tres actividades más vinculadas al proceso de evaluación formativa: (1) una coevaluación intergrupal de las preguntas, que ofrece feedback sobre las preguntas diseñadas por otros grupos, (2) una tutorización del docente, que mantendrá varias reuniones formativas con los grupos antes de la calificación final. Además, se desarrolla (3) un proceso de autoevaluación/coevaluación intergrupal del diseño de las preguntas a través de una escala de valoración (ver Anexo 1). Finalmente, el profesor recopilará las preguntas en un documento a modo de batería.

Así se entra en la segunda tarea de la propuesta, en la que los alumnos, en posesión de la batería de preguntas, inician el proceso de preparación del examen. Si bien se trata de un momento evidentemente individual, se mantiene la organización de

\footnotetext{
${ }^{1}$ Siguiendo unas pautas aportadas por el profesor al inicio de la propuesta, y que determina el número y formato de las preguntas, el tipo de preguntas, el formato de entrega, etc.
}

La evaluación formativa y compartida desde un enfoque competencial. Aplicación práctica en tareas de aula en Primaria y Secundaria 
grupos y se plantean dos actividades (estrategias de aula) para promover una mejora de los vínculos y ayudas entre los alumnos: (a) mediante la estrategia cooperativa de marcador colectivo ${ }^{2}$, y (b) repaso de los contenidos en forma de concurso grupal ${ }^{3}$.

Por último, la tercera tarea se desarrolla primero con una prueba individual, aplicándose posteriormente una actividad que implica a los alumnos en la corrección (autoevaluación y coevaluación). Esto permite al alumnado ser conscientes de aspectos competenciales relacionados con la valoración del trabajo y del esfuerzo ${ }^{4}$.

En el Anexo I se incluye un cuadro-resumen del proceso de evaluación formativa y su vinculación con la calificación.

\section{Propuesta 2: Búsqueda documental colaborativa + trabajo de síntesis de $\mathbf{1 0 0 0}$ palabras}

Esta experiencia relaciona los "proyectos de trabajo" (Hernández, 1988, 2002) con un Ambiente Virtual de Aprendizaje (AVA) ${ }^{5}$. Así, partiendo de una propuesta general de contenidos por parte del profesor, se concreta junto a los alumnos la temática definitiva en base a sus intereses y preferencias. A partir de la selección del tema, se plantea de forma consensuada la pregunta temática o guía ${ }^{6}$, y derivado de ésta se define el producto final de aprendizaje ${ }^{7}$. Para la consecución del producto final de aprendizaje, que se lleva a cabo de manera colaborativa, se planifican cuatro tareas de carácter competencial asociadas a procesos de evaluación formativa compartida (ver resumen en Anexo II):

(1) Búsqueda, recopilación y análisis documental colaborativo, organizado en microgrupos y haciendo uso de una plantilla editada previamente por el profesor en Google-docs (Pérez-Pueyo, Heras-Bernardino \& Hortigüela, 2014).

\footnotetext{
2 Por ejemplo: "si conseguís que todos los miembros del grupo alcancen una nota superior al cinco, todos aumentaréis dos puntos la calificación".

${ }^{3}$ Los equipos se irán lanzando preguntas entre sí, de forma que, si consiguen llegar entre todos los grupos a un número de preguntas acertadas (previamente establecido), conseguirán todos una puntuación extra en la calificación final.

${ }^{4}$ Estos aprendizajes competenciales se incluyen en la secuenciación de las competencias para aprender a aprender y a la competencia de autonomía e iniciativa personal (Proyecto INCOBA).

${ }^{5}$ En este caso, entendemos por AVA el espacio en el que tiene lugar una educación virtual.

${ }^{6}$ Un ejemplo de pregunta temática es la siguiente: ¿Cómo podemos dar a conocer información relativa a la actividad física en edad temprana con el mayor número de receptores posible?

7 "La creación de una píldora formativa e-learning, su incorporación a un canal de youtube y posterior difusión mediante redes sociales: Facebook y Twiter".
} 
(2) Trabajo de sintesis de 1000 palabras, realizado a partir de la documentación recopilada. Se mantiene la misma organización de los microgrupos, y versa sobre una de las temáticas establecidas desde el inicio del proyecto.

(3) Creación y edición de un videotutorial o "píldora formativa e-learning". Se establecen dos posibles alternativas: la realización de un "screencast" a través de una presentación (power-point o prezzi) asociada a una narración en audio, o la realización de un video-tutorial en la que los alumnos aparezcan en imagen haciendo una presentación del trabajo realizado.

(4) Difusión de la píldora formativa, haciendo uso fundamentalmente de redes sociales.

\section{Síntesis}

A lo largo del taller se muestran dos tareas de carácter competencial, en las que la participación activa del alumno, la ayuda entre iguales y la evaluación formativa y compartida se convierten en estrategias clave para la construcción de los aprendizajes y como parte de la competencia en aprender a aprender.

\section{Referencias}

Heras-Bernardino, C., \& Pérez-Pueyo, A. (2017). El proceso de evaluación formativa en la etapa de bachillerato: trabajo de síntesis a partir de una búsqueda documental colaborativa. En V. López-Pastor \& A. Pérez-Pueyo (coords.), Evaluación formativa y compartida en educación: experiencias de éxito en todas las etapas educativas (pp. 272-285). León: Universidad de León

Hernández, F. (1988). La globalización mediante proyectos de trabajo. Cuadernos de pedagogía, 155, 54-59.

Hernández, F. (2002). Los proyectos de trabajo. Mapa para navegantes en mares de incertidumbre. Cuadernos de pedagogía, 310, 78-82

Lozano, C., Casado. O. M., \& Pérez-Pueyo, Á. (20017). La preparación de exámenes a través de la evaluación formativa y la redacción colectiva de preguntas y respuestas. En V. López-Pastor \& Á. Pérez-Pueyo (coords.), Evaluación formativa y compartida en educación: experiencias de éxito en todas las etapas educativas. (pp. 272-285). León: Universidad de León 
Pérez-Pueyo, A. (coord.) (2013). Programar y evaluar competencias básicas en 15 pasos. Barcelona: Graó.

Pérez-Pueyo, A., Heras, C. \& Casado, O. (2014). Instrumento de Calificación: Escala de valoración "Trabajo de síntesis de 1000 palabras" (heteroevaluación y coevaluación). Recuperado de https://drive.google.com/file/d/0B7xEdP1O4dj8WG02MmpMbi1tdjA/view

Pérez-Pueyo, A., Heras, C. \& Hortigüela, D. (2014). Plantilla para la Búsqueda colaborativa de referencias para el Trabajo de Síntesis de 1000 palabras. Recuperado de https://drive.google.com/file/d/0B7xEdP1O4dj8UlVUb3dCMkZlWEU/view

Pérez-Pueyo, A., Heras, C. \& Hortigüela, D. (2015). Instrumento de Calificación: Escala de valoración de la búsqueda colaborativa de referencias (heteroevaluación $\quad \mathrm{y}$ coevaluación). Recuperado de https://drive.google.com/file/d/0B7xEdP1O4dj8ZXZoQjB3QzkzWE0/view

Pérez-Pueyo, A. \& Casado, O. (2015). Escala graduada para la valoración del videotutorial. Recuperado de https://rive.google.com/file/d/0B7xEdP1O4dj8ZzBjb0xEeV96dE0/view 


\section{Anexos}

Anexo 1: Tabla-Resumen del proceso de evaluación formativa (propuesta: diseño de preguntas de examen)

\begin{tabular}{|c|c|c|c|c|c|}
\hline Competencias clave & $\begin{array}{l}\text { Actividades } \\
\text { aprendizaje }\end{array}$ & Evaluación formativa & Instrumentos & $\%$ & Requisitos \\
\hline $\begin{array}{l}\text { Competencias sociales y } \\
\text { cívicas } \\
\text { - Trabajo grupal }\end{array}$ & \multirow{4}{*}{$\begin{array}{l}\text { Redacción y } \\
\text { presentación } \\
\text { de preguntas } \\
\text { de examen }\end{array}$} & $\begin{array}{l}\text { Feedback del profesor y } \\
\text { de los compañeros } \\
\text { (coevaluación informal) } \\
\text { durante el proceso }\end{array}$ & $\begin{array}{l}\text { Plantilla tipo } \\
\text { de preguntas }\end{array}$ & ----- & \multirow{10}{*}{$\begin{array}{l}\text { No se aceptarán } \\
\text { entregas de } \\
\text { tareas que no } \\
\text { cumplan con los } \\
\text { plazos y la } \\
\text { forma } \\
\text { establecida }\end{array}$} \\
\hline $\begin{array}{l}\text { Competencia Lingüística: } \\
\text { - Expresión escrita } \\
\text { - Vocabulario }\end{array}$ & & $\begin{array}{l}\text { Autoevaluación y } \\
\text { coevaluación de la } \\
\text { producción final } \\
\text { (preguntas de examen) }\end{array}$ & $\begin{array}{l}\text { Escala de } \\
\text { valoración }\end{array}$ & $10 \%$ & \\
\hline $\begin{array}{l}\text { - Comprensión lectora } \\
\text { Competencia en aprender a } \\
\text { aprender } \\
\text { - Autoevaluación } \\
\text { - Coevaluación }\end{array}$ & & $\begin{array}{l}\text { Heteroevaluación de la } \\
\text { producción final } \\
\text { (preguntas de examen): } \\
\text { entregas parciales y final }\end{array}$ & \multirow{2}{*}{$\begin{array}{l}\text { Escala de } \\
\text { valoración }\end{array}$} & \multirow{2}{*}{$35 \%$} & \\
\hline $\begin{array}{l}\text { Competencia Sentido de la } \\
\text { iniciativa y espíritu } \\
\text { emprendedor } \\
\text { - Organización del } \\
\text { trabajo en grupo }\end{array}$ & & $\begin{array}{l}\text { Autoevaluación } \\
\text { intragrupal diaria durante } \\
\text { el proceso de elaboración } \\
\text { de las preguntas }\end{array}$ & & & \\
\hline \multirow{4}{*}{$\begin{array}{l}\text { Competencia en aprender a } \\
\text { aprender } \\
\text { - Autoevaluación } \\
\text { Competencias sociales y } \\
\text { cívicas } \\
\text { - Trabajo grupal } \\
\text { Competencia Sentido de la } \\
\text { iniciativa y espíritu } \\
\text { emprendedor } \\
\text { - Organización del } \\
\text { trabajo individual y } \\
\text { grupal }\end{array}$} & \multirow{4}{*}{$\begin{array}{l}\text { Preparación } \\
\text { del examen }\end{array}$} & $\begin{array}{l}\text { Feedback de compañeros } \\
\text { y profesor durante la } \\
\text { preparación del examen }\end{array}$ & --------------- & ------ & \\
\hline & & $\begin{array}{l}\text { Análisis de las preguntas } \\
\text { (profesor y alumnos) } \\
\text { durante el concurso } \\
\text { intergrupal }\end{array}$ & -------------- & ------ & \\
\hline & & $\begin{array}{l}\text { Autoevaluación } \\
\text { intragrupal diaria durante } \\
\text { el proceso de preparación }\end{array}$ & DSI & \multirow{2}{*}{$15 \%$} & \\
\hline & & $\begin{array}{l}\text { Heteroevaluación del } \\
\text { funcionamiento de los } \\
\text { grupos }\end{array}$ & $\begin{array}{l}\text { Escala de } \\
\text { valoración }\end{array}$ & & \\
\hline \multirow{2}{*}{$\begin{array}{l}\text { Competencia en aprender a } \\
\text { aprender } \\
\text { - Autoevaluación } \\
\text { - Coevaluación }\end{array}$} & \multirow{2}{*}{$\begin{array}{l}\text { Desarrollo de } \\
\text { la prueba } \\
\text { escrita } \\
\text { individual }\end{array}$} & $\begin{array}{l}\text { Implicación del alumno } \\
\text { en la corrección: } \\
\text { Autoevaluación y } \\
\text { Coevaluación }\end{array}$ & $\begin{array}{l}\text { Escala de } \\
\text { valoración }\end{array}$ & ------ & \\
\hline & & Heteroevaluación & $\begin{array}{l}\text { Escala de } \\
\text { valoración }\end{array}$ & $40 \%$ & \\
\hline
\end{tabular}


Anexo 2: Tabla-Resumen del proceso de evaluación formativa (propuesta: búsqueda documental + trabajo de síntesis)

\begin{tabular}{|c|c|c|c|c|c|}
\hline Competencias Clave & $\begin{array}{l}\text { Actividades } \\
\text { Aprendizaje }\end{array}$ & Evaluación formativa & $\begin{array}{l}\text { Instrumentos } \\
\text { calificación }\end{array}$ & $\%$ & Requisitos \\
\hline \multirow{4}{*}{$\begin{array}{l}\text { Competencia Sentido de la } \\
\text { iniciativa y espíritu } \\
\text { emprendedor } \\
\text { - Búsqueda de información } \\
\text { - Autonomía (participación en } \\
\text { proyectos) } \\
\text { - Organización del trabajo } \\
\text { Competencias sociales y cívicas } \\
\text { - Trabajo grupal } \\
\text { Competencia digital } \\
\text { - Seguridad TIC } \\
\text { Competencia en aprender a } \\
\text { aprender: } \\
\text { - Autoevaluación } \\
\text { - Coevaluación }\end{array}$} & \multirow{4}{*}{$\begin{array}{l}\text { Búsqueda y } \\
\text { análisis } \\
\text { documental } \\
\text { colaborativa }\end{array}$} & Foros en el AVA & & & \multirow{13}{*}{$\begin{array}{l}\text { No se } \\
\text { aceptarán } \\
\text { entregas de } \\
\text { tareas que no } \\
\text { cumplan con } \\
\text { los plazos y } \\
\text { la forma } \\
\text { establecida }\end{array}$} \\
\hline & & $\begin{array}{l}\text { Revisión continua y } \\
\text { feedback de las } \\
\text { aportaciones en plantilla } \\
\text { googledocs }\end{array}$ & & & \\
\hline & & $\begin{array}{l}\text { Heteroevaluación } \\
\text { producción final: se aporta } \\
\text { información mediante } \\
\text { escala de valoración }\end{array}$ & $\begin{array}{l}\text { Escala de } \\
\text { valoración }^{8}\end{array}$ & $18 \%$ & \\
\hline & & $\begin{array}{l}\text { Coevaluación intergrupal + } \\
\text { autoevaluación grupal del } \\
\text { producto final }\end{array}$ & $\begin{array}{l}\text { Escala de } \\
\text { valoración }\end{array}$ & $12 \%$ & \\
\hline \multirow{4}{*}{$\begin{array}{l}\text { Competencia Lingüística: } \\
\text { - Expresión escrita } \\
\text { Competencias sociales y cívicas } \\
\text { - Trabajo grupal } \\
\text { Competencia en aprender a } \\
\text { aprender: } \\
\text { - Autoevaluación } \\
\text { - Coevaluación }\end{array}$} & \multirow{4}{*}{$\begin{array}{l}\text { Trabajo de } \\
\text { síntesis de } \\
1000 \\
\text { palabras }\end{array}$} & Foros en el AVA & --------------- & & \\
\hline & & $\begin{array}{l}\text { Revisión y feedback de las } \\
\text { dos entregas parciales } \\
\text { obligatorias. }\end{array}$ & --------------- & & \\
\hline & & $\begin{array}{l}\text { Heteroevaluación de la } \\
\text { producción final: se aporta } \\
\text { información mediante } \\
\text { escala de valoración }\end{array}$ & $\begin{array}{l}\text { Escala de } \\
\text { valoración }{ }^{9}\end{array}$ & $24 \%$ & \\
\hline & & $\begin{array}{l}\text { Coevaluación intergrupal + } \\
\text { autoevaluación grupal del } \\
\text { producto final }\end{array}$ & $\begin{array}{l}\text { Escala de } \\
\text { valoración }\end{array}$ & $16 \%$ & \\
\hline \multirow{4}{*}{$\begin{array}{l}\text { Competencia Sentido de la } \\
\text { iniciativa y espíritu } \\
\text { emprendedor } \\
\text { - Organización del trabajo } \\
\text { Competencia Lingüística: } \\
\text { - Expresión escrita } \\
\text { Competencia en aprender a } \\
\text { aprender: } \\
\text { - Coevaluación }\end{array}$} & \multirow{4}{*}{$\begin{array}{l}\text { Creación y } \\
\text { edición de } \\
\text { una "píldora } \\
\text { formativa e- } \\
\text { learning" }\end{array}$} & Foros en el AVA & --------------- & & \\
\hline & & $\begin{array}{l}\text { Revisión y feedback de las } \\
\text { entregas parciales a través } \\
\text { del AVA }\end{array}$ & & & \\
\hline & & $\begin{array}{l}\text { Heteroevaluación de la } \\
\text { producción final: se aporta } \\
\text { información mediante } \\
\text { escala de valoración }\end{array}$ & $\begin{array}{l}\text { Escala } \\
\text { graduada }^{10}\end{array}$ & $18 \%$ & \\
\hline & & $\begin{array}{l}\text { Coevaluación intergrupal } \\
\text { del producto final }\end{array}$ & $\begin{array}{l}\text { Escala } \\
\text { graduada }\end{array}$ & $12 \%$ & \\
\hline $\begin{array}{l}\text { Competencias sociales y cívicas } \\
\text { - Trabajo grupal } \\
\text { - Mejora social } \\
\text { Competencia digital } \\
\text { - Comunicación (redes } \\
\text { sociales) }\end{array}$ & $\begin{array}{l}\text { Difusión de } \\
\text { la "píldora }\end{array}$ & $\begin{array}{l}\text { Seguimiento de la difusión } \\
\text { en redes sociales }\end{array}$ & & & \\
\hline
\end{tabular}

\footnotetext{
${ }^{8}$ Puede consultarse en Pérez-Pueyo, Heras, Hortigüela (2015).

${ }^{9}$ Puede consultarse en Pérez-Pueyo, Heras, Casado (2014).

${ }^{10}$ Puede consultarse en Pérez-Pueyo \& Casad (2015).
}

La evaluación formativa y compartida desde un enfoque competencial. Aplicación práctica en tareas de aula en Primaria y Secundaria 